\title{
A Maternal Dietary Pattern High in Discretionary Foods was Inversely Associated with Psychomotor Development of Infants at 1 Year ${ }^{\dagger}$
}

\author{
Jamie de Seymour 1,* , Kathryn Beck ${ }^{1}$, Cathryn Conlon ${ }^{1}$, Mary Beatrix Jones ${ }^{2}$, John Colombo ${ }^{3}$, \\ Yin-Yin Xia ${ }^{4}$, Ting-Li Han ${ }^{5}$, Hong-Bo Qi ${ }^{5}$, Hua Zhang ${ }^{5}$ and Philip Baker ${ }^{6}$ \\ 1 College of Health, Massey University, Auckland 0632, New Zealand; k.1.Beck@massey.ac.nz (K.B.); \\ c.conlon@massey.ac.nz (C.C.) \\ 2 Department of Statistics, University of Auckland, Auckland 1010, New Zealand; \\ beatrix.jones@auckland.ac.nz \\ 3 Department of Psychology and Schiefelbusch Institute for Life Span Studies, University of Kansas, \\ Lawrence, KS 66045, USA; colombo@ku.edu \\ 4 School of Public Health and Management, Chongqing Medical University, Chongqing 400016, China; \\ kendraxia@163.com \\ 5 Department of Obstetrics and Gynaecology, The First Affiliated Hospital of Chongqing Medical \\ University, Chongqing 400016, China; t.han@auckland.ac.nz (T.-L.H.); qihongbo@sina.com (H.-B.Q.); \\ zh2844@gmail.com (H.Z.) \\ 6 College of Life Sciences, University of Leicester, Leicester LE1 7RH, UK; philip.baker@leicester.ac.uk \\ * Correspondence: j.deseymour@massey.ac.nz; Tel.: +64-(09)-414-0800 (ext. 43660) \\ + Presented at the 2019 Annual Meeting of the Nutrition Society of New Zealand, Napier, New Zealand, 28- \\ 29 November 2019.
}

Published: 16 December 2019

A plethora of studies have investigated the relationship between maternal dietary intake and infant's neurocognitive development. However, these studies have predominantly focused on individual nutrients or food groups and findings from randomised controlled trials have been inconsistent. In humans, nutrients are consumed in foods, which are eaten in combinations. One way to assess dietary intake within the context it is consumed, is to use dietary pattern analysis. Our study aimed to use a dietary pattern approach to investigate the relationship between maternal diet and infant's neurocognitive development.

Dietary intake was assessed in 974 pregnant women from China, using a 110-item food frequency questionnaire. The food groups were condensed into 28 groups according to nutrient profiles. Principal component analysis followed by orthogonal varimax rotation was performed on the condensed food groups, to derive dietary patterns. The Chinese-adapted Bayley Scales of Infant Development was used to assess neurodevelopment of infants at 1 year of age. Each infant received a standardised score on the psychomotor development index (PDI) and mental development index (MDI). Multivariate linear regression was used to assess associations between index scores and dietary pattern scores, adjusting for a comprehensive set of socio-demographic and health related confounders.

Two main dietary patterns were consumed by this cohort; a "Prudent" diet high in vegetables, fruit, poultry, fish, seafood, organ meats, eggs, nuts, dairy and bread and a "Discretionary foods" diet high in oils and condiments, fast foods, sweetened beverages, and pasta. After adjustment for confounding variables, there was a significant inverse relationship between maternal scores on the "Discretionary foods" pattern and infant's scores on the PDI $(\mathrm{P}=0.04, \beta(95 \% \mathrm{CI})=-1.22(-2.37$, $-0.06)$ ). 
The next stage of this research involves metabolomic analysis to explore whether variations in scores on the "Discretionary foods" pattern are reflected in the maternal metabolome.

(C) 2019 by the authors. Licensee MDPI, Basel, Switzerland. This article is an open access article distributed under the terms and conditions of the Creative Commons Attribution (CC BY) license (http://creativecommons.org/licenses/by/4.0/). 